\title{
Exact computation of the Voronoi Diagram of spheres in 3D, its topology and its geometric invariants
}

\author{
Anton, François; Mioc, Darka; Santos, Marcelo
}

Published in:

Proceedings of the 8th ISVD International Symposium on Voronoi Diagrams in Science and Engineering

Link to article, DOI:

10.1109/ISVD.2011.16

Publication date:

2011

Link back to DTU Orbit

Citation (APA):

Anton, F., Mioc, D., \& Santos, M. (2011). Exact computation of the Voronoi Diagram of spheres in 3D, its topology and its geometric invariants. In Proceedings of the 8th ISVD International Symposium on Voronoi Diagrams in Science and Engineering (pp. 58-66). https://doi.org/10.1109/ISVD.2011.16

\section{General rights}

Copyright and moral rights for the publications made accessible in the public portal are retained by the authors and/or other copyright owners and it is a condition of accessing publications that users recognise and abide by the legal requirements associated with these rights.

- Users may download and print one copy of any publication from the public portal for the purpose of private study or research.

- You may not further distribute the material or use it for any profit-making activity or commercial gain

- You may freely distribute the URL identifying the publication in the public portal 


\section{Exact computation of the Voronoi Diagram of spheres in 3D, its topology and its geometric invariants}

\author{
François Anton \\ Department of Informatics and Mathematical Modeling \\ Technical University of Denmark \\ Kongens Lyngby, Denmark \\ Email: fa@imm.dtu.dk
}

\author{
Darka Mioc \\ National Space Institute \\ Technical University of Denmark \\ Kongens Lyngby, Denmark \\ Email: mioc@space.dtu.dk
}

\author{
Marcelo Santos \\ Department of Geodesy and Geomatics Engineering \\ University of New Brunswick \\ Fredericton, New Brunswick, Canada \\ Email: santos@unb.ca
}

\begin{abstract}
In this paper, we are addressing the exact computation of the Delaunay graph (or quasi-triangulation) and the Voronoi diagram of spheres using Wu's algorithm. Our main contribution is first a methodology for automated derivation of invariants of the Delaunay empty circumcircle predicate for spheres and the Voronoi vertex of four spheres, then the application of this methodology to get all geometrical invariants that intervene in this problem and the exact computation of the Delaunay graph and the Voronoi diagram of spheres. To the best of our knowledge, there does not exist a comprehensive treatment of the exact computation with geometrical invariants of the Delaunay graph and the Voronoi diagram of spheres. Starting from the system of equations defining the zero-dimensional algebraic set of the problem, we are following Wu's algorithm to transform the initial system into an equivalent Wu characteristic (triangular) set. In the corresponding system of algebraic equations, in each polynomial (except the first one), the variable with higher order from the preceding polynomial has been eliminated (by pseudo-remainder computations) and the last polynomial is a polynomial of a single variable. By regrouping all the formal coefficients for each monomial in each polynomial, we get polynomials that are invariants for the given problem. We rewrite the original system by replacing the invariant polynomials by new formal coefficients. We repeat the process until all the algebraic relationships (syzygies) between the invariants have been found by applying Wu's algorithm on the invariants.
\end{abstract}

\section{Keywords}

Voronoi diagram of spheres, Delaunay graph of spheres, Wu's method, invariants, characteristic set, ascending chains, algebraico-differential ideals

\section{Introduction}

Voronoi diagrams have been a central topic in research in computational geometry since their inception [13], [7], [4], [5], [15], and they have many applications in different scientific and engineering disciplines [25], [17]. However, generalized Voronoi diagrams, and especially the Voronoi diagram of spheres have not been explored sufficiently
[21]. With recent scientific discoveries in biology and chemistry, Voronoi diagrams of spheres have become more important for representing and analysing the molecular 3D structure and surface [27], the structure of the protein [19], etc.

Will [32] provides the method of the computation of additively weighted Voronoi Cells for applications in molecullar Biology. His algorithm is based on the the general methods for computing lower envelopes of algebraic surfaces [32], and it does not provide efficient method for updating the topology of the Voronoi diagrams of spheres. In his algorithm he has to maintain three kinds of conflicts associated with the vertices, edge fragments and face fragments.

Gavrilova provided an early work on generalised Voronoi diagrams in her doctoral thesis [11] and subsequent work by Gavrilova and Rokne on topology updating of the kinematic Voronoi diagram of hyperspheres [10]. Gavrilova was the first one to provide an explicit algorithm for the computation of the vertices of the Voronoi diagram of spheres [12], which uses Cramer's rule to solve $x, y$ and $z$ as affine functions of $v$, and then replace $x, y$ and $z$ by their functions of $v$ to get a quadratic equation in $v$, that can be solved exactly using radicals, thus getting an exact computation of the Voronoi vertices. The degree of their predicate for hyperspheres in $d$-dimensional space was $2(d+1)$ in the variables defining the spheres, thus 8 in the case of spheres in $3 D$. We have found the formulas analogous to formulas (7), (8), (9) and (10) included in [10] automatically using Wu's algorithm: they constitute the Ritt-Wu's characteristic set for the polynomial set corresponding to the Voronoi vertex of four spheres. Gavrilova and Rokne do not actually state explicitely that these were invariants nor their geometric interpretation nor all their algebraic relationships (syzygies or rewriting rules). 
Nishida and Sugihara [23] and Nishida et al. [24] extended the results in [10] by providing the topological structure of the Voronoi diagram of hyperspheres in $d$-dimensional space using low precision arithmetic. They prove that they need only $2 d+4$ times longer bits for exact computation than the bits used for the input in [23]. They exhibit in the formulas (38), (39), (40) and (41) in [23] linear relations between the grouping of terms in the function they evaluate in floating-point arithmetic. However, they do not actually state explicitely that these were invariants nor their geometric interpretation nor all their non-linear algebraic relationships (syzygies or rewriting rules).

Kim et al. provide several important research contributions in the domain of the Voronoi diagrams of spheres including one patent [18] for the computation of three-dimensional (3D) Voronoi diagrams. Their work provides many new algorithms related to the Voronoi diagrams of which the most relevant to this topic are the computation of three-dimensional (3D) Voronoi diagrams [18], Euclidean Voronoi diagram of 3D balls and its computation via tracing edges [17] and the Euclidean Voronoi diagrams of 3D spheres and applications to protein structure analysis [19].

Recently, Hanniel and Elber [16] provide an algorithm for computation of the Voronoi diagrams for planes, spheres and cylinders in $\mathbb{R}^{3}$. Their algorithm is based on computing the lower envelope of the bisector surfaces similar to the algorithm of Will [32]. However, all of the current research efforts did not provide the exact method for the Voronoi Diagram and Delaunay graph (or quasi-triangulation) of spheres. The dual graph of the Voronoi diagram, which Anton [2], [3] names "Delaunay graph" was later named "quasitriangulation" and studied in Kim et al. [28], [20]. Anton and Mioc have provided an exact method for the computation of the Voronoi diagram of circles [1] using Gröbner basis and invariants. This paper provides a generalisation to the three-dimensional case using a much more powerful (working with differential polynomials) and tractable method: Wu's method [33].

The exact knowledge of the Delaunay graph for curved objects may sound like a purely theoretical knowledge that is not central in practical applications. This is not always the case in some applications. These applications include material science, metallography, spatial analyses and VLSI layout. The Johnson-Mehl tessellations (which generalise several weighted Voronoi diagrams) [26] play a central role in the Kolmogorov-Johnson-Mehl-Avrami [22] nucleation and growth kinetics theory. The Kolmogorov theory provides an exact description of the kinetics during the heating and cooling processes in material science (the Kolmogorov equation [22]). The exact knowledge of the neighbourliness among molecules is central to the prediction of the formation of particle aggregates. In metallography, the analysis of precipitate sizes in aluminium alloys through Transmission Electronic Microscopy [9, Section 1.2.2] provides an exact measurement of the cross sections of these precipitates when they are "rodes" with a fixed number of orientations [9, Section 1.2.2]. In VLSI design, the second order Voronoi diagram of the layout is used in the computation of the critical area, a measure of a circuit layout's sensitivity to spot defects [8, Section 1]. An important concern on critical area computation is robustness $[8$, Section 1].

Another limitation of approximative algorithms for the computation of the Delaunay graph is that when approximate computations are performed on objects defined approximately (within some geometric tolerance), the propagation of the errors can be critical, especially if the final computation involves approximate intermediary computations. Finally, the exact computation of the Delaunay graph participates to the recent move in the development of numerical and simulation software as well as computer algebra systems to exact systems [6]. In this paper, we present a simplification of the expression of both the Voronoi vertex of spheres and the empty sphere criterion as well as a novel method of computation of the Voronoi Diagram and Delaunay graph of spheres and their invariants using Wu's method.

This paper is organised as follows. In Section 2, we review the preliminaries: the Voronoi diagram and Delaunay graph of spheres. In Section 3, we present Wu's method. In Sections 4 and 5, we present a simplification of the expression of both the Voronoi vertex of spheres and the empty sphere criterion as well as a novel method of computation of the Voronoi Diagram and Delaunay graph of spheres and their invariants using Wu's method. Section 4 is devoted to the Voronoi vertex while Section 5 is devoted to the Delaunay empty circumsphere criterion. Finally, in Section 6, we conclude the paper.

\section{Preliminaries}

Voronoi diagrams are irregular tessellations of space, where space is continuous and structured by discrete objects [26]. The Voronoi tessellation of a set of sites is a decomposition of the space into proximal regions (one for each site). Sites were points for the first historical Voronoi diagrams [29], [30], [31], but in this paper we will explore sets of spheres. The proximal region corresponding to one site (i.e. its Voronoi region) is the set of points of the space that are closer to that site than to any other site of the set of sites [26]. We will recall now the formal definitions of the Voronoi diagram and of the Delaunay graph. For this purpose, we need to recall some basic definitions.

Definition 2.1. (Metric) Let $M$ be an arbitrary set. A metric on $M$ is a mapping $d: M \times M \rightarrow \mathbb{R}_{+}$such that for any 
elements $a, b$, and $c$ of $M$, the following conditions are fulfilled: $d(a, b)=0 \Leftrightarrow a=b, d(a, b)=d(b, a)$, and $d(a, c) \leq d(a, b)+d(b, c) .(M, d)$ is then called a metric space, and $d(a, b)$ is the distance between $a$ and $b$.

Remark 2.2. The Euclidean space $\mathbb{R}^{N}$ with the Euclidean distance $\delta$ is a metric space $\left(\mathbb{R}^{N}, \delta\right)$.

Let $M=\mathbb{R}^{N}$, and $\delta$ denote a distance between points. Let $\mathcal{S}=\left\{s_{1}, \ldots, s_{m}\right\} \subset M, m \geq 2$ be a set of $m$ different subsets of $M$, which we call sites. The distance between a point $x$ and a site $s_{i} \subset M$ is defined as $d\left(x, s_{i}\right)=\inf _{y \in s_{i}}\{\delta(x, y)\}$.

Definition 2.3. (Bisector) For $s_{i}, s_{j} \in \mathcal{S}, s_{i} \neq s_{j}$, the bisector $B\left(s_{i}, s_{j}\right)$ of $s_{i}$ with respect to $s_{j}$ is: $B\left(s_{i}, s_{j}\right)=\{x \in$ $\left.M \mid d\left(x, s_{i}\right)=d\left(x, s_{j}\right)\right\}$.

Definition 2.4. (Influence zone) For $s_{i}, s_{j} \in \mathcal{S}, s_{i} \neq s_{j}$, the influence zone $D\left(s_{i}, s_{j}\right)$ of $s_{i}$ with respect to $s_{j}$ is: $D\left(s_{i}, s_{j}\right)=\left\{x \in M \mid d\left(x, s_{i}\right)<d\left(x, s_{j}\right)\right\}$.

Definition 2.5. (Voronoi region) The Voronoi region $V\left(s_{i}, \mathcal{S}\right)$ of $s_{i} \in \mathcal{S}$ with respect to the set $\mathcal{S}$ is: $V\left(s_{i}, \mathcal{S}\right)=$ $\bigcap_{s_{j} \in \mathcal{S}, s_{j} \neq s_{i}} D\left(s_{i}, s_{j}\right)$.

Definition 2.6. (Voronoi diagram) The Voronoi diagram of $\mathcal{S}$ is the union $V(\mathcal{S})=\bigcup_{s_{i} \in \mathcal{S}} \partial V\left(s_{i}, \mathcal{S}\right)$ of all region boundaries.

Definition 2.7. (Delaunay graph) The Delaunay graph $D G(\mathcal{S})$ of $\mathcal{S}$ is the dual graph of $V(\mathcal{S})$ defined as follows:

- the set of vertices of $D G(\mathcal{S})$ is $\mathcal{S}$,

- for each $(N-1)$-dimensional facet of $V(\mathcal{S})$ that belongs to the common boundary of $V\left(s_{i}, \mathcal{S}\right)$ and of $V\left(s_{j}, \mathcal{S}\right)$ with $s_{i}, s_{j} \in \mathcal{S}$ and $s_{i} \neq s_{j}$, there is an edge of $D G(\mathcal{S})$ between $s_{i}$ and $s_{j}$ and reciprocally, and

- for each vertex of $V(\mathcal{S})$ that belongs to the common boundary of $V\left(s_{i_{1}}, \mathcal{S}\right), \ldots, V\left(s_{i_{N+2}}, \mathcal{S}\right)$, with $\forall k \in$ $\{1, \ldots, N+2\}, s_{i_{k}} \in \mathcal{S}$ all distinct, there exists a complete graph $K_{N+2}$ between the $s_{i_{k}}, k \in\{1, \ldots, N+2\}$, and reciprocally.

The one-dimensional elements of the Voronoi diagram are called Voronoi edges. The points of intersection of the Voronoi edges are called Voronoi vertices. The Voronoi vertices are points that have at least $N+1$ nearest neighbours among the sites of $\mathcal{S}$. In the plane, the Voronoi diagram forms a network of vertices and edges. In the plane, when sites are points in general position, the Delaunay graph is a triangulation known as the Delaunay triangulation. In the plane, the Delaunay graph satisfies the following empty sphere criterion: no site intersects the interior of the spheres touching (tangent to without intersecting the interior of) the sites that are the vertices of any tetrahedron of the Delaunay graph.

Once the Voronoi region a query point belongs to has been identified, it is easy to answer proximity queries. The closest site from the query point is the site whose Voronoi region is the Voronoi region that has been identified. The Voronoi diagram defines a neighbourhood relationship among sites: two sites are neighbours if, and only if, their Voronoi regions are adjacent, or alternatively, there exists an edge between them in the Delaunay graph.

The exact computation of the Delaunay graph is important for two reasons. By exact computation, we mean a computation whose output is correct. First, unlike the Voronoi diagram, the Delaunay graph is a discrete structure, and thus it does not lend itself to approximations. Second, the inaccurate computation of this Delaunay graph can induce inconsistencies within this graph, which may cause a program that updates this graph to crash. This is particularly true for the randomised incremental algorithm for the construction of the Voronoi diagram of spheres. In order to maintain the Delaunay graph after each addition of a sphere, we need to detect the Delaunay tetrahedra that are not empty any longer, and we need to detect which new tetrahedra formed with the new sphere are empty, and thus valid. This handles both old vertices that should not be kept in the new Voronoi diagram, as well as old edges that should be shrunk (leading to new Voronoi vertices), as well as old facets that should be shrunk (leading also to new Voronoi vertices). The algorithm that certifies whether the tetrahedron formed by 4 given spheres is empty (i.e. the sphere tangent to the 4 given spheres does not contain any point of a given sphere in its interior) or not empty is used for checking which old tetrahedra are not empty any longer and which new tetrahedra formed with the new sphere are empty, and thus valid. This algorithm is called the "Delaunay graph conflict locator" in the reminder of this paper.

The new tetrahedra that are checked are either defined by two spheres being linked by an existing Delaunay edge, or three spheres b. Its output is the list of all the Voronoi vertices corresponding to the 1 -dimensional facets of the Delaunay graph having the first 3 spheres as vertices whose circumspheres contain a point of the fourth sphere in their interior, and a value that certifies the presence of each Voronoi vertex in that list. The fact that a circumsphere (the sphere that is externally tangent to three given spheres) is not empty is equivalent to the tetrahedron formed by those three spheres being not Delaunay, and this is called a conflict. Thus, it justifies the name of "Delaunay graph conflict locator". In the context of the ordinary Voronoi diagram of points in the plane, the concept that is analogous to the Delaunay graph conflict locator is the Delaunay graph predicate, which certifies whether a tetrahedron of the Delaunay triangulation is such that its circumsphere does not contain a given point.

\section{Wu's algorithm}

Let $\mathcal{K}=\mathbb{Q}$ be the field of rational numbers, $\mathbb{X}=\left\{x_{1}, \cdots, x_{n}\right\}$ a set of variables, $\mathcal{K}[\mathbb{X}]$ be the set 
of polynomials in the variables of $\mathbb{X}$, with coefficients in $\mathcal{K}$. If not otherwise stated the order of the monomials composing a polynomial will be taken as the lexicographic monomial ordering where $1 \prec x_{1} \prec x_{2} \prec \cdots \prec x_{n}$. Let $P \in \mathcal{K}[\mathbb{X}]$ be a polynomial. The universal field $\mathcal{E}$ over $\mathcal{K}$ is an algebraically closed field containing an infinite number of indeterminates, or more simply a projective space over an algebraically closed field.

Definition 3.1. (from [33]) For any set of polynomials $\mathbb{P} \subset$ $\mathcal{K}[\mathbb{X}), \operatorname{Zero}(\mathcal{P})=\left\{x \in \mathcal{E}^{n} \mid P(x)=0, \forall P \in \mathbb{P}\right\}$ is called a variety. For a set of polynomials $\mathbb{P}$ and a polynomial $D$, we define $Z \operatorname{ero}(\mathbb{P} / D)=Z \operatorname{ero}(\mathbb{P}) \backslash Z \operatorname{ero}(\{D\})$, called a quasi-algebraic variety.

The aim of Wu's method is to determine the decomposition of quasi-algebraic varieties into irreducible components and the dimensions of these irreducible components. For example Zero $(x y)$ in the three-dimensional projective space corresponds to the union of two irreducible components of dimension 2: the projective plane Zero $(x)$ and the projective plane Zero $(y)$. Likewise, Wu's method allowed us to prove that the generalized $\epsilon$-offset (i.e. the set of centers of spheres of radius $\epsilon$ that are tangent) to a sphere of centre $\Pi$ and of radius $r$ is the union of two irreducible components of dimension 2: the sphere of center $\Pi$ and of radius $r+\epsilon$, and the sphere of center $\Pi$ and of radius $|r-\epsilon|$.

Definition 3.2. (from [33]) Let $P \in k[x]$ be a polynomial. The class of $P$, denoted by $\operatorname{cls}(P)$ is the $c$ such that $x_{c}$ is the largest variable that occurs in $P$. If $c l s(P)=c$, then $x_{c}$ is called the leading variable and denoted by $\operatorname{lvar}(P)$, the highest degree monomial of $P$ as a univariate polynomial in lvar $(P)$ is called the leading monomial, and its coefficient is called the initial of $P$.

Typically, the polynomial $D$ whose variety is subtracted from a polynomial set will be a product of initials corresponding to geometric invariants that correspond to special cases of the geometric problem at hand.

Definition 3.3. (from [33]) A polynomial $P_{1}$ has higher ordering than a polynomial $P_{2}$, denoted as $P_{2} \prec P_{1}$, if either $\operatorname{cls}\left(P_{1}\right)>\operatorname{cls}\left(P_{2}\right)$, or $c=\operatorname{cls}\left(P_{1}\right)=\operatorname{cls}\left(P_{2}\right)$ and $\operatorname{deg}\left(P_{1}, x_{c}\right)>\operatorname{deg}\left(P_{2}, x_{c}\right)$. If none of two polynomials has higher ordering than the other, they are said to have the same rank, denoted as $P_{1} \sim P_{2}$.

Definition 3.4. (from [33]) A polynomial $Q$ is reduced with respect to $P$, if $\operatorname{cls}(P)=c>0$ and $\operatorname{deg}\left(Q, x_{c}\right)<$ $\operatorname{deg}\left(P, x_{c}\right)$. A sequence of non-zero polynomials $\mathcal{A}$ : $A_{1}, A_{2}, \cdots, A_{r}$ is a triangular set if either $r=1$ or cls $\left(A_{1}\right)<\operatorname{cls}\left(A_{2}\right)<\cdots<\operatorname{cls}\left(A_{r}\right)$. A triangular set is called an ascending chain, or simply a chain, if $A_{j}$ is reduced with respect to $A_{i}$ for $i<j$. For a chain $\mathcal{A}$, we denote $\mathbb{I}_{\mathcal{A}}$ as the product of the initials of the polynomials in $\mathcal{A}$.
Definition 3.5. (from [33]) Let $\mathcal{A}^{\prime}: A_{1}^{\prime}, A_{2}^{\prime}, \cdots, A_{r^{\prime}}^{\prime}$ and $\mathcal{A}^{\prime \prime}$ : $A_{1}^{\prime \prime}, A_{2}^{\prime \prime}, \cdots, A_{r^{\prime \prime}}^{\prime \prime}$ be two (ascending) chains. $\mathcal{A}^{\prime}$ is said to be of lower ordering than $\mathcal{A}^{\prime \prime}$, denoted as $\mathcal{A}^{\prime} \prec \mathcal{A}^{\prime \prime}$, if either there is some $k$ such that $A_{1}^{\prime} \sim A_{1}^{\prime \prime} \cdots A_{k-1}^{\prime} \sim A_{k-1}^{\prime \prime}$, while $A_{k}^{\prime} \prec A_{k}^{\prime \prime}$; or $r^{\prime}>r^{\prime \prime}$ and $A_{1}^{\prime} \sim A_{1}^{\prime \prime} \cdots A_{r^{\prime \prime}}^{\prime \prime} \sim A_{r^{\prime \prime}}^{\prime \prime}$.

Lemma 3.6. (from [33]) A sequence of (ascending) chains steadily lower in ordering is finite.

Definition 3.7. (from [33]) A basic set of a polynomial set $\mathbb{P}$ is any chain of lowest ordering contained in $\mathbb{P}$. A polynomial $Q$ is called reduced with respect to a chain $\mathcal{A}$ if $Q$ is reduced with respect to all the polynomials in $\mathcal{A}$.

Lemma 3.8. (from [33]) Let $\mathcal{A}$ be a basic set of a polynomial set $\mathbb{P}$. If $P$ is reduced with respect to $\mathcal{A}$, then a basic set of $\mathbb{P} \cup P$ is of lower ordering than that of $\mathbb{P}$.

Let $F$ and $G$ be non-zero polynomials with $c=\operatorname{cls}(F)$ and $I=\operatorname{init}(F)$. Either $G$ is reduced with respect to $F$ (which means that $\operatorname{deg}\left(G, x_{c}\right)<\operatorname{deg}\left(F, x_{c}\right)$ ), or $\operatorname{deg}\left(G, x_{c}\right) \geq \operatorname{deg}\left(F, x_{c}\right)$, and then it is possible to divide $G$ by $F$ as univariate polynomials in $x_{c}$. Indeed, let $k=\operatorname{deg}\left(G, x_{c}\right)-\operatorname{deg}\left(F, x_{c}\right), k^{\prime}=\operatorname{deg}\left(G, x_{c}\right)$, and $I^{\prime}$ be the coefficient of $x_{c}^{k^{\prime}}$ in $G$, then $\operatorname{deg}\left(I G-I^{\prime} x_{c}^{k} F\right)<k^{\prime}$. Therefore in a finite number of steps $s \leq k+1$, we get that $I^{s} G=Q F+R$ where $Q$ and $R$ are polynomials in $\mathcal{K}[\mathbb{X}]$ with $R$ reduced with respect to $F$. $R$ is uniquely determined and called the reminder of $G$ with respect to $F$ and denoted as $R=\operatorname{prem}(G, F)$.

It is therefore trivial to generalize this Euclidean division to the case of a triangular system: the result of the division of a polynomial $G$ with respect to the polynomials of the triangular set $\mathcal{A}: A_{1}, A_{2}, \cdots, A_{r}$ is obtained by repeated division of $G$ by $A_{1}, A_{2}, \cdots, A_{r}$. Thus, we get the division formula: $J G=\sum_{i} Q_{i} A_{i}+R$, where $R$ is reduced with respect to all the polynomials in the triangular set $\mathcal{A}$, and $R$ is called the reminder of $G$ with respect to $\mathcal{A}$, and denoted as $R=\operatorname{prem}(G, \mathcal{A})$ [33].

This leads to Wu's algorithm for producing the decomposition of a variety into irreducible varieties (corresponding to irreducible polynomial sets). Starting with a polynomial set $\mathbb{P}_{0}=\mathbb{P}$, one should select a basis $\mathcal{B}_{0}$ of $\mathbb{P}_{0}$, and then compute the set $\mathbb{R}_{0}$ of non-zero reminders of polynomials of $\mathbb{P}_{0} \backslash \mathcal{B}_{0}$ with respect to $\mathcal{B}_{0}$. Then, let $\mathbb{P}_{1}=\mathbb{P}_{0} \cup \mathbb{R}_{0}$. Then, one should compute a basis set $\mathbb{B}_{1}$ in $\mathbb{P}_{1}$. By Lemma $3.8, \mathcal{B}_{1}$ is of lower ordering than $\mathcal{B}_{0}$. Then, one should compute the set $\mathbb{R}_{1}$ of non-zero reminders of polynomials of $\mathbb{P}_{1} \backslash \mathcal{B}_{1}$ with respect to $\mathcal{B}_{1}$. Therefore, such a process has a finite number of steps, and the final result is a basic set $\mathcal{B}_{m}=\mathcal{C}$, such that the corresponding set of non-zero reminders $\mathbb{R}_{m}$ is the empty set and $\operatorname{prem}(\mathbb{P}, \mathcal{C})=\{0\}$. Thus, for each chain that can be obtained in such a way, $\operatorname{Zero}(\mathbb{P}) \subseteq \operatorname{Zero}(\mathcal{C})$. Thus, one can obtain the variety of $\mathbb{P}$ as a union of quasi-projective varieties, where each algebraic variety is irreducible and the algebraic varieties 
being subtracted correspond to degenerate cases expressed as geometric invariants. Any chain $\mathcal{C}$ obtained by applying Wu's algorithm is called a characteristic set [33].

\section{The Voronoi vertex of four spheres}

This is the first part of our contribution: the exact computation of the Voronoi vertices of four spheres and of their invariants as well as the algebraic relationships between their invariants (also called syzygies). For this purpose we systematically replace the formal coefficient of each monomial as new variables (that may correspond to geometric invariants). The initials of each polynomial appearing as quotient of a division is in fact an invariant of the geometric problem, since it conditions the degree of the corresponding algebraic variety and its irreducibility. In the reminder of the paper, we will denote any sphere either by its name or its name followed in parentheses by its center and its radius (e.g. $S_{1}((a, b, c), r$,$) denotes the sphere centered at$ $(a, b, c$,$) and of radius r$. A true Voronoi vertex of four spheres $S_{1}((a, b, c), r),, S_{2}((d, e, f), s),, S_{3}((g, h, i), t$,$) ,$ and $S_{4}((j, k, l), u$,$) is the intersection of four generalized$ $v$-offsets to $S_{1}, S_{2}, S_{3}$, and $S_{4}$ (see Figure 4.1). This is indeed the case because even if a point is in the interior of the disk bounded by a sphere (i.e. inside the sphere but not on the sphere), it has a positive distance with respect to the sphere. Therefore, we need to consider both positive offsets (expansions) of spheres and negative offsets (contractions) of spheres in the equations of the generalized offsets of spheres.

Therefore, a Voronoi vertex is a solution of one of the following system $\mathcal{I}$ of polynomial equations:

$$
\mathcal{I}:\left\{\begin{array}{l}
(x-a)^{2}+(y-b)^{2}+(z-c)^{2}-(r \pm v)^{2}=0 \\
(x-d)^{2}+(y-e)^{2}+(z-f)^{2}-(s \pm v)^{2}=0 \\
(x-g)^{2}+(y-h)^{2}+(z-i)^{2}-(t \pm v)^{2}=0 \\
(x-j)^{2}+(y-k)^{2}+(z-l)^{2}-(u \pm v)^{2}=0
\end{array}\right.
$$

We can set the origin of the coordinate system at the center of the smallest sphere among $S_{1}, S_{2}, S_{3}$, and $S_{4}$, and subtract its radius $r$ from the radii of the other spheres (assume without loss of generality that the first sphere was the sphere with smallest radius). This simplification has been obtained automatically using Wu's method: the differences of radii and of coordinates of the centers of the spheres appear in the basic set of the polynomial set without simplification. Since the generalized $v$-offset of a sphere centered on $(a, b, c)$ and of radius $s$ is the union of two concentric spheres centered on $(a, b, c)$ and of radii $s+v$ and $|s-v|$, we can state that a Voronoi vertex is a zero of one of the following polynomial sets (i.e. a point on which all the polynomials in one of the following polynomial sets $\mathcal{I I}$ evaluate to 0 ):

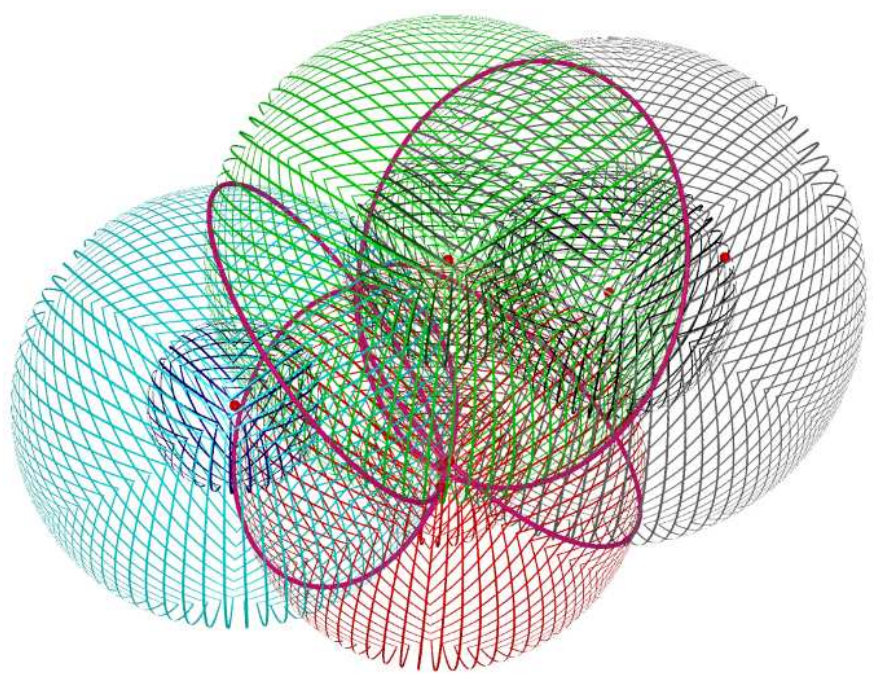

Fig. 4.1. A Voronoi vertex of four spheres as the intersection point of four sphere generalized offset irreducible components. The circles that are drawn are circles of intersection of two spheres.

$$
\mathcal{I} \mathcal{I}:\left\{\begin{array}{c}
x^{2}+y^{2}+z^{2}-(v)^{2} \\
\left(x-a^{\prime}\right)^{2}+\left(y-b^{\prime}\right)^{2}+\left(z-c^{\prime}\right)^{2}-\left(s^{\prime} \pm v\right)^{2} \\
\left(x-d^{\prime}\right)^{2}+\left(y-e^{\prime}\right)^{2}+\left(z-f^{\prime}\right)^{2}-\left(t^{\prime} \pm v\right)^{2} \\
\left(x-g^{\prime}\right)^{2}+\left(y-h^{\prime}\right)^{2}+\left(z-i^{\prime}\right)^{2}-\left(u^{\prime} \pm v\right)^{2}
\end{array}\right.
$$

where $\left(a^{\prime}, b^{\prime}, c^{\prime}\right)=(d, e, f)-(a, b, c)$, $\left(d^{\prime}, e^{\prime}, f^{\prime}\right)=(g, h, i)-(a, b, c),\left(g^{\prime}, h^{\prime}, i^{\prime}\right)=(j, k, l)-(a, b, c)$, $s^{\prime}=s-r, t^{\prime}=t-r$ and $u^{\prime}=u-r$.

This simplification shows that the apparent 16 possible configuration cases of four spheres (corresponding to the 16 possible cases of system $\mathcal{I}$ ) are not linearly independent, but pair up two by two, since the nature of the irreducible component of the first generalized offset (expansion or retraction) does not have any influence on the polynomial sets $\mathcal{I} \mathcal{I}$ whose common roots are the Voronoi vertices of the four spheres. Moreover, the same basic set shows that by subtracting the equation of the first generalized offset from the equations of the second, third and fourth generalized offsets, we get an equivalent polynomial set composed of a single quadratic polynomial $s_{1}$ of a sphere generalized offset and three linear polynomials $p_{1}, p_{2}$ and $p_{3}$ :

$$
\left\{\begin{array}{c}
x^{2}+y^{2}+z^{2}-(v)^{2} \\
-2 a^{\prime} x-2 b^{\prime} y-2 c^{\prime} z-2 s^{\prime} v+\left(a^{\prime 2}+b^{\prime 2}+c^{\prime 2}-s^{\prime 2}\right) \\
-2 d^{\prime} x-2 e^{\prime} y-2 f^{\prime} z-2 t^{\prime} v+\left(d^{\prime 2}+e^{\prime 2}+f^{\prime 2}-t^{\prime 2}\right) \\
-2 g^{\prime} x-2 h^{\prime} y-2 i^{\prime} z-2 u^{\prime} v+\left(g^{\prime 2}+h^{\prime 2}+i^{\prime 2}-u^{\prime 2}\right)
\end{array}\right.
$$

In these systems, all polynomials have the same class: if we assume a variable ordering $v \prec x \prec y \prec z$, all the polynomials above have $z$ has higher ordering variable (i.e. class) and their degree in the class is 2 for $s_{1}$ and 1 for $p_{1}$, $p_{2}$, and $p_{3}$. Thus, $s_{1}$ has a higher ordering than $p_{1}, p_{2}$, and $p_{3}$. Therefore, it is possible to divide $s_{1}, p_{1}, p_{2}$, or $p_{3}$ by 
$p_{1}, p_{2}$, or $p_{3}$. We start with $\mathbb{P}_{0}=\left\{s_{1}, p_{1}, p_{2}, p_{3}\right\}$ and an ascending chain $\mathcal{A}_{0}=p_{1}$. An ascending chain is obtained from $\mathbb{P}_{0}$ by repeated division of the polynomials that are not already in the ascending chain, adding all non-zero reminders to the polynomial set $\mathbb{P}_{0}$, and adding the non-zero reminder with lowest ordering to the chain $\mathcal{A}_{0}$. The reminders of the division by $\mathcal{A}_{0}$ are polynomials in $v, x$, and $y$. The first reminder that has the lowest ordering is $r_{00}=\operatorname{prem}\left(p_{2}, p_{1}\right)$. It can be added to $\mathcal{A}_{0}$. However, $r_{01}=\operatorname{prem}\left(p_{3}, p_{1}\right)$ and $r_{02}=\operatorname{prem}\left(s_{1}, p_{1}\right)$ cannot be added to $\mathcal{A}_{0}$, since they have the same ordering as $r_{00}$ (same class and same degree). The next non-zero reminder that is added to the chain $\mathcal{A}_{1}$ is $r_{10}=\operatorname{prem}\left(r_{01}, r 00\right)$, which is a polynomial in $v$ and $x$. The other non-zero reminder at this class level is another polynomial in $v$ and $x: r_{11}=\operatorname{prem}\left(r_{02}, r_{00}\right)$. We obtain a basis set when the lowest ordering non-zero reminder is a single-valued polynomial. In this case, this is achieved after the following non-zero reminder is computed: $r_{20}=\operatorname{prem}\left(r_{11}, r_{10}\right)$, which is a quadratic polynomial in $v$. However, from all the computed non-zero reminders, we select polynomials with fewest monomials. Thus, our basic set is $\mathcal{C}: C_{1}, C_{2}, C_{3}, C_{4}$, where

$$
\left\{\begin{array}{c}
C_{1}=J \cdot v^{2}+K \cdot v+L \\
C_{2}=A \cdot x+H \cdot v+I \\
C_{3}=-A \cdot y+E \cdot v+F \\
C_{4}=A \cdot z+B \cdot v+C .
\end{array}\right.
$$

It is a characteristic set of $\mathbb{P}$, since the class is strictly increasing along the ascending chain, and every polynomial $C_{j}$ occurring after a polynomial $C_{i}$ (with $j>i$ ) is reduced with respect of $C_{j}$. Since the univariate polynomial is of degree 2 in $v$ all the reminders of polynomials by $\mathcal{A}$ will be univariate polynomials of degree at most 1 in $v$. The offset variable can be computed by solving the quadratic equation $J \cdot v^{2}+K \cdot v+L=0$, which has no solution if $K^{2}<4 J L$, one solution $v=\frac{K}{2 J}$ if $K^{2}=4 J L$, and two solutions $v=\frac{K \pm \sqrt{K^{2}-4 J L}}{2 J}$ if $K^{2}>4 J L$. In the case where the original polynomial set corresponds to expansions of spheres, the coefficients of the monomials of the polynomials in the preceding basic set are:

$$
\begin{aligned}
& A=-\left|\begin{array}{lll}
a^{\prime} & b^{\prime} & c^{\prime} \\
d^{\prime} & e^{\prime} & f^{\prime} \\
g^{\prime} & h^{\prime} & i^{\prime}
\end{array}\right| \\
& B=2\left|\begin{array}{lll}
a^{\prime} & b^{\prime} & -s^{\prime} \\
d^{\prime} & e^{\prime} & -t^{\prime} \\
g^{\prime} & h^{\prime} & -u^{\prime}
\end{array}\right| \\
& C=\left|\begin{array}{lll}
a^{\prime} & b^{\prime} & a^{\prime 2}+b^{\prime 2}+c^{2}-s^{2} \\
d^{\prime} & e^{\prime} & d^{\prime 2}+e^{\prime 2}+f^{\prime 2}-t^{\prime 2} \\
g^{\prime} & h^{\prime} & g^{\prime 2}+h^{\prime 2}+i^{\prime 2}-u^{\prime 2}
\end{array}\right| \\
& E=2\left|\begin{array}{lll}
a^{\prime} & c^{\prime} & -s^{\prime} \\
d^{\prime} & f^{\prime} & -t^{\prime} \\
g^{\prime} & i^{\prime} & -u^{\prime}
\end{array}\right|
\end{aligned}
$$

$$
\begin{aligned}
& F=\left|\begin{array}{lll}
a^{\prime} & c^{\prime} & a^{\prime 2}+b^{\prime 2}+c^{2}-s^{2} \\
d^{\prime} & f^{\prime} & d^{\prime 2}+e^{\prime 2}+f^{\prime 2}-t^{\prime 2} \\
g^{\prime} & i^{\prime} & g^{\prime 2}+h^{2}+i^{\prime 2}-u^{\prime 2}
\end{array}\right| \\
& H=2\left|\begin{array}{lll}
b^{\prime} & c^{\prime} & -s^{\prime} \\
e^{\prime} & f^{\prime} & -t^{\prime} \\
h^{\prime} & i^{\prime} & -u^{\prime}
\end{array}\right| \\
& I=\left|\begin{array}{lll}
b^{\prime} & c^{\prime} & a^{\prime 2}+b^{\prime 2}+c^{\prime 2}-s^{\prime 2} \\
e^{\prime} & f^{\prime} & d^{\prime 2}+e^{\prime 2}+f^{\prime 2}-t^{\prime 2} \\
h^{\prime} & i^{\prime} & g^{\prime 2}+h^{\prime 2}+i^{\prime 2}-u^{\prime 2}
\end{array}\right|
\end{aligned}
$$

The seven other cases can be computed likewise. By the division formula, $E$ can be obtained from $B$ by exchanging the column $\left(\begin{array}{c}b^{\prime} \\ e^{\prime} \\ h^{\prime}\end{array}\right)$ with the column $\left(\begin{array}{c}c^{\prime} \\ f^{\prime} \\ i^{\prime}\end{array}\right)$, and $H$ can be obtained from $E$ by exchanging the column $\left(\begin{array}{c}a^{\prime} \\ d^{\prime} \\ g^{\prime}\end{array}\right)$ with the column $\left(\begin{array}{c}b^{\prime} \\ e^{\prime} \\ h^{\prime}\end{array}\right)$. The same rewriting rules allow one to convert $C$ into $F$ and $F$ into $I$ respectively.

We can recognize that $A$ is minus the signed volume of the tetrahedron formed by the four sphere centers, which is invariant by any positive isometry (i.e. any geometric transformation that preserves distances and oriented angles, which can be expressed as a composition of translations and rotations). It is therefore a geometric invariant (i.e. a quantity that does not change even if we apply a geometric transformation to all the four spheres defining a Voronoi vertex), and depending on it being zero or not, there are either one or zero Voronoi vertices or two Voronoi vertices. The formal coefficient $B$ is also a geometric invariant, since it is the signed volume of a tetrahedra, where the first three points are the first three sphere centers, and the last point coordinates are the differences of radii between the second, third and fourth spheres with respect to the first sphere. This is again invariant by any positive isometry. The formal coefficient $C$ is the signed volume of a tetrahedra, where the first three points are the first three sphere centers, and the last point coordinates with respect to the center of the first sphere are the powers of the center of the first sphere with respect to the second, third and fourth spheres. This is again invariant by any positive isometry.

Applying the above mentioned rewriting rules, the formal coefficient $E$ is the signed volume of a tetrahedra, where the first three points are the first, second and fourth sphere centers, and the last point coordinates are the differences of radii between the second, third and fourth spheres with respect to the first sphere. This is again invariant by any positive isometry. The formal coefficient $F$ is the signed volume of a tetrahedra, where the first three points are the first, second 
and fourth sphere centers, and the last point coordinates with respect to the center of the first sphere are the powers of the center of the first sphere with respect to the second, third and fourth spheres. This is again invariant by any positive isometry.

Again applying the above mentioned rewriting rules, the formal coefficient $H$ is the signed volume of a tetrahedra, where the first three points are the first, third and fourth sphere centers, and the last point coordinates are the differences of radii between the second, third and fourth spheres with respect to the first sphere. This is again invariant by any positive isometry. The formal coefficient $H$ is the signed volume of a tetrahedra, where the first three points are the first, third and fourth sphere centers, and the last point coordinates with respect to the center of the first sphere are the powers of the center of the first sphere with respect to the second, third and fourth spheres. This is again invariant by any isometry. Therefore, $A, B, C, D, E, F, G, H$ and $I$ are geometric invariants (by any isometry) of the Voronoi vertex of four spheres.

These results could not have been obtained from a Gröbner basis of the polynomial set of all the coefficients of the polynomials in $\mathcal{C}$ with variables being the invariants $A, B, C, D, E, F, G, H, I, J, K, L$. Attempting the Gröbner basis on an Apple Mac Pro server with 6GB of RAM using the computer algebra system Singular (see [14] for an introduction to Singular) gives a "no more memory" error message after 14510 new polynomials have been added to the Gröbner basis. Wu's method is more powerful and tractable, because it does not require a basis that is composed of polynomials of the polynomial set, whose leading monomials generate the set of leading monomials of all the polynomials in the polynomial set, but requires only a basic set that is composed of polynomials that form a chain of lowest ordering in the polynomial set. Therefore, Wu's method involves fewer divisions, because it does not need to prove that the leading monomial of any polynomial of the polynomial set (also called ideal) can be expressed as a polynomial combination of the leading monomials of the polynomials in the Gröbner basis. Finally, Wu's method provides a constructive method by pseudo-reminders, that is more predictable and tractable, because one can compute a bound on the number of steps beforehand.

Using these simple invariants, it is possible by repeated division to get the reminder of the invariants corresponding to monomials of $C_{1}$ by a basic set of the polynomial set corresponding to the simple invariants. We get the following simplified expressions for the coefficients of the monomials of the univariate polynomial in $v$ :

$\left\{\begin{array}{c}J=B^{2}+E^{2}-A^{2}+H^{2} \\ K=2 B C+2 E F+2 H I \\ L=C^{2}+F^{2}+I^{2}\end{array}\right.$

We can interpret geometrically $J$ as the power of a point $P 1$ at the extremity of the vector $(B, E, H)$ placed at the center of $S_{1}$ with respect to a sphere centered on the center of $S_{1}$ and of radius $A$. It is also possible to interpret geometrically $L$ as the square norm of a vector $V 1=(C, F, I)$. Finally, $K$ can be interpreted geometrically as the scalar product of the position vector of $P 1$ with respect to the center of $S_{1}$ and the vector $V 1$. Therefore $J, K$ and $L$ are also geometric invariants, that are unchanged by any positive isometry. Notice that all the geometric invariants correspond to geometric quantities that are unchanged either by positive isometries: signed volumes, or by all isometries: norms and scalar products. However, applying a negative isometry (that preserves the distances but inverts the oriented angles) induces changes of signs of all the terms in each one of the polynomials $C_{2}, C_{3}$, and $C_{4}$ of the basic set $\mathcal{A}$. Therefore the corresponding algebraic variety is the same, showing that the Voronoi diagram is preserved by any isometry. All these results have been confirmed automatically using Wu's algorithm by introducing manually all the geometric invariants corresponding to signed volumes of tetrahedra and scalar products of vectors.

Using geometric invariants represents a very important simplification by using invariants. Indeed, we have rewritten in a quadratic univariate polynomial in $v$ of the form $J \cdot v^{2}+K \cdot v+L$ the term in $v^{2}$, that had 224 monomials in the parameters $a, b, c, d, e, f, g, h, i, s, t, u$ into a term $J$ that has only 4 monomials in the simple invariants mentioned above. Moreover, we have rewritten the term in $v$, that had 1080 monomials in the parameters $a, b, c, d, e, f, g, h, i, s, t, u$ into a term $K$ that has only 3 monomials in the simple invariants mentioned above. Finally, we have rewritten the constant term, that had 2276 monomials in the parameters $a, b, c, d, e, f, g, h, i, s, t, u$ into a term $L$ that has only 3 monomials in the simple invariants mentioned above. The univariate polynomial of the ascending chain has thus been simplified from a polynomial containing 3580 terms into a polynomial containing only 10 terms using invariants!

\section{Delaunay empty circumsphere predicate for spheres}

In order to evaluate the Delaunay empty circumsphere predicate for spheres, we need to compute whether the distance between the Voronoi vertex of $S_{1}, S_{2}, S_{3}$, and $S_{4}$ with coordinates $\left(\begin{array}{lll}x & y & z\end{array}\right)$ with respect to the center of $S_{1}$ and the fifth sphere $s_{5}$ with center having coordinates $\left(\begin{array}{lll}j & k & l\end{array}\right)$ with respect to the center of $S_{1}$ and radius $m+r$ is lower than the (common) distance between $\left(\begin{array}{lll}x & y & z\end{array}\right)$ and $S_{1}, S_{2}, S_{3}$, and $S_{4}$. However, there are two possible position configurations of the fifth sphere with respect to the Voronoi vertex $\left(\begin{array}{lll}x & y & z\end{array}\right)$ : either the fifth sphere does not contain $\left(\begin{array}{lll}x & y & z\end{array}\right)$ or the fifth sphere contains $\left(\begin{array}{lll}x & y & z\end{array}\right)$ (see Figures 5.1 and 5.2). The polynomial stating the difference of squared distances between the Voronoi vertex and the fifth sphere 


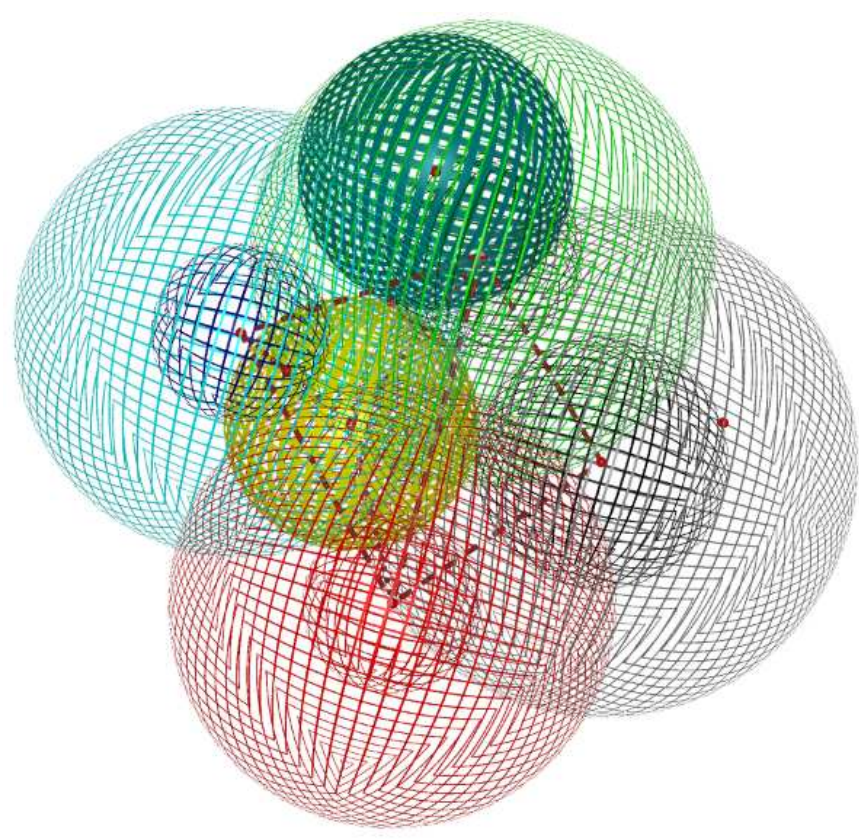

Fig. 5.1. The Voronoi vertex is at the same distance with respect to its four defining spheres (represented as small hatches) and a fifth sphere (represented by large hatches) that does not contain the Voronoi vertex. The circumsphere tangent to the four defining spheres is also represented by large hatches.

and between the Voronoi vertex and $S_{1}, S_{2}, S_{3}$, and $S_{4}$ is $G=(x-j)^{2}+(y-k)^{2}+(z-l)^{2}-(m \pm v)^{2}$. Using repeated division of $G$ with respect to the polynomials of the triangular set $\mathcal{C}$, we get the following univariate (in $v$ ) reminders of $G$ by the ascending chain $\mathcal{A}$ :

For the Voronoi vertex exterior to the fifth sphere, we get: $G=2\left(-m+\frac{j H-k E+l B}{A}\right) \cdot v+$

$\left(\frac{K}{J}+2 \frac{B C+E F+H I}{A^{2}}-\frac{\left(B^{2}+E^{2}+H^{2}\right) K}{A^{2} J}\right) \cdot v+$

$\left(j^{2}+k^{2}+l^{2}-m^{2}\right)+2 \frac{j I-k F+l C}{A}+\frac{L}{J}+$

$\frac{C^{2}+F^{2}+I^{2}}{A^{2}}-\frac{\left(B^{2}+E^{2}+H^{2}\right) L}{A^{2} J}$

For the Voronoi vertex interior to the fifth sphere, we get: $G=2\left(m+\frac{j H-k E+l B}{A}\right) \cdot v+$

$\left(\frac{K}{J}+2 \frac{B C+E F+H I}{A^{2}}-\frac{\left(B^{2}+E^{2}+H^{2}\right) K}{A^{2} J}\right) \cdot v+$

$\left(j^{2}+k^{2}+l^{2}-m^{2}\right)+2 \frac{j I-k F+l C}{A}+\frac{L}{J}+$

$\frac{C^{2}+F^{2}+I^{2}}{A^{2}}-\frac{\left(B^{2}+E^{2}+H^{2}\right) L}{A^{2} J}$

We can therefore compute exactly the Delaunay empty circumsphere predicate for spheres exactly from the exact value of $v$. By the division formula, the last expression (for the Voronoi vertex being inside the fifth sphere) of $G$ can be obtained from the former expression (for the Voronoi vertex being outside the fifth sphere) of $G$ by the following rewriting

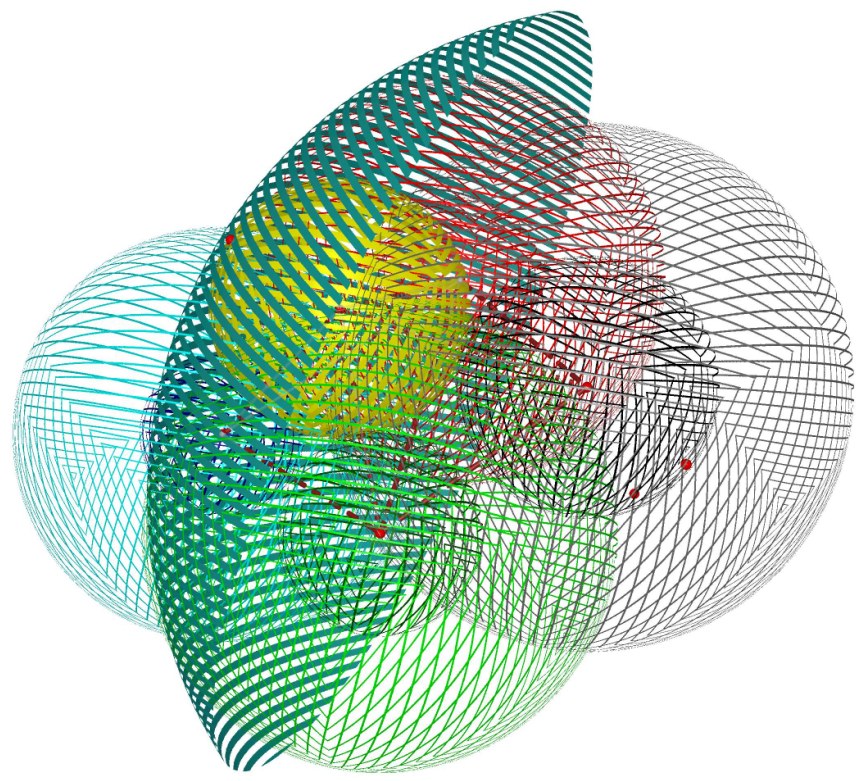

Fig. 5.2. The Voronoi vertex is at the same distance with respect to its four defining spheres (represented as small hatches) and a fifth sphere (represented by large hatches) that does not contain the Voronoi vertex. The circumsphere tangent to the four defining spheres is also represented by large hatches.

rule: $m$ must be replaced by $-m$, which is logical since the only term in $m$ is a term in $m v$, and subtracting the offset instead of adding it corresponds exactly to this rewriting rule.

The degree of the Delaunay graph empty circumsphere predicate characterizes together with the number of monomials the algebraic complexity of this predicate, which evaluates the sign of $G$. We have already seen the dramatic simplification of the polynomial defining the offset parameter $v$ in the previous section (10 terms instead of 3580). Finally, we conclude on the degree of the predicate and the precision necessary to compute exactly the results in floating point arithmetic.

Proposition 5.1. The algebraic degree of the incircle predicate for spheres in the invariants and the variables defining the fifth sphere is 6 . We need 6 times longer bits for the exact computation of the incircle predicate than the bits used for the invariants and the variables defining the fifth sphere.

Proof: The incircle predicate is given by the sign of $G$. Since the denominator of $v$ is $2 J$, the greatest common divider of all the terms in the expansion of $G$ is $A^{2} J^{2}$, which is either positive or zero. In the generic case $\left(A^{2} J^{2} \neq 0\right)$, we can rewrite $G$ as a rational function, where the denominator is $A^{2} J^{2}$. Thus, the sign of $G$ is determined only by the sign of the numerator of the preceding rational function. We can see immediately that the degree of this numerator in the invariants 
and the variables defining the fifth sphere is the degree of the monomials $m K A^{2} J^{2}$ or $\left(j^{2}+k^{2}+l^{2}-m^{2}\right) A^{2} J^{2}$, which is 6 . Bounding all the invariants and the variables defining the fifth sphere as in [23], we get that we need 6 times longer bits for the exact computation of the incircle predicate than the bits used for the invariants and the variables defining the fifth sphere.

\section{Conclusions}

This research work provides a significative simplification using invariants for the exact computation of vertices of the Voronoi diagram of spheres and the empty circumsphere criterion as well as their geometric invariants. This work has a direct application in Geodesy: the optimal placement of the system of GPS satellites and the determination of the geometric uncertainty of the determination of coordinates by GPS as a function of the placement of the GPS satellites. Further work will address these applications as well as the application of the automatic derivation and simplification of invariants to the Delaunay graph and Voronoi diagram of quadrics.

\section{References}

[1] François Anton, Darka Mioc, and Christopher Gold, The voronoi diagram of circles and its application to the visualization of the growth of particles, Transactions on Computational Science III (Marina L. Gavrilova and C. J. Tan, eds.), Springer-Verlag, Berlin, Heidelberg, 2009, pp. 20-54.

[2] Francois Anton, Voronoi diagrams of semi-algebraic sets, $\mathrm{Ph} . \mathrm{D}$. thesis, The University of British Columbia (UBC), Vancouver, Canada, 2004.

[3] Francois Anton, A certified delaunay graph conflict locator for semialgebraic sets, Computational Science and Its Applications - ICCSA 2005, International Conference, Singapore, May 9-12, 2005, Proceedings, Part I, 2005, pp. 669-682.

[4] F. Aurenhammer, Gewichtete Voronoi Diagramme: Geometrische Deutung und Konstruktions-Algorithmen, Ph.D. thesis, IIG-TU Graz, Austria, 1984, Report B53.

[5] F. Aurenhammer and R. Klein, Voronoi diagrams, Handbook of Computational Geometry, Chapter V (J. Sack and G. Urrutia, eds.), Elsevier Science Publishing, 2000, pp. 201-290.

[6] Lenore Blum, Felipe Cucker, Michael Shub, and Steve Smale, Complexity and real computation, Springer-Verlag, New York, 1998, With a foreword by Richard M. Karp. MR 99a:68070

[7] K. Q. Brown, Voronoi diagrams from convex hulls, Information Processing Letters 9 (1979), no. 5, 223-228.

[8] Zhenming Chen, Evanthia Papadopoulou, and Jinhui Xu, Robust algorithm for k-gon voronoi diagram construction, Abstracts for the Fourteenth Canadian Conference on Computational Geometry CCCG '02 (Lethbridge, Alberta, Canada), University of Lethbridge, August 2002, pp. 77-81.

[9] Alexis Deschamps, Handbook of aluminum, ch. Analytical Techniques for Aluminium Alloys, vol. 2, Alloy Production and Materials manufacturing, pp. 155-192, Marcel Dekker, Inc., New York, USA, 2005.

[10] M. L. Gavrilova and J. Rokne, Updating the topology of the dynamic voronoi diagram for spheres in euclidean d-dimensional space, Comput. Aided Geom. Des. 20 (2003), 231-242.

[11] Marina Gavrilova, Proximity and applications in general metrics, Ph.D. thesis, University of Calgary, Calgary, Alberta, Canada, 1998.

[12] Marina L. Gavrilova, An explicit solution for computing the vertices of the euclidean d-dimensional voronoi diagram of spheres in a floatingpoint arithmetic, International Journal of Computational Geometry and Applications 19 (2009), 415-424.

[13] P.J. Green and R. Sibson, Computing dirichlet tessellations in the plane, The Computer Journal 21 (1977), no. 2, 168-173.
[14] Gert-Martin Greuel and Gerhard Pfister, A singular introduction to commutative algebra, Springer-Verlag, Berlin, 2002, With contributions by Olaf Bachmann, Christoph Lossen and Hans Schönemann, With 1 CD-ROM (Windows, Macintosh, and UNIX). MR 1930604

[15] Leonidas J. Guibas, Donald E. Knuth, and Micha Sharir, Randomized incremental construction of delaunay and voronoi diagrams, ICALP '90: Proceedings of the 17th International Colloquium on Automata, Languages and Programming (London, UK), Springer-Verlag, 1990, pp. $414-431$.

[16] Iddo Hanniel and Gershon Elber, Computing the voronoi cells of planes, spheres and cylinders in $\mathbb{R}^{3}$, Comput. Aided Geom. Des. 26 (2009), 695-710.

[17] Deok-Soo Kim, Youngsong Cho, and Donguk Kim, Euclidean voronoi diagram of $3 d$ balls and its computation via tracing edges., ComputerAided Design (2005), 1412-1424.

[18] Deok-soo Kim, Youngsong Cho, and Donguk Kim, Calculating threedimensional (3d) voronoi diagrams, Patent no. 7825927, November 2010.

[19] Deok-Soo Kim, Youngsong Cho, Donguk Kim, Sangsoo Kim, Jonghwa Bhak, and Sung-Hoon Lee, Euclidean voronoi diagrams of $3 d$ spheres and applications to protein structure analysis, Japan Journal of Industrial and Applied Mathematics 22 (2005), 251-265, 10.1007/BF03167441.

[20] Deok-Soo Kim, Youngsong Cho, and Kokichi Sugihara, Quasi-worlds and quasi-operators on quasi-triangulations, Comput. Aided Des. 42 (2010), 874-888.

[21] Donguk Kim and Deok-Soo Kim, Region-expansion for the voronoi diagram of $3 d$ spheres, Comput. Aided Des. 38 (2006), 417-430.

[22] A. Kolmogorov, A statistical theory for the recrystallization of metals, Akad. nauk SSSR, Izv., Ser. Matem. (1937), no. 3, 355-359.

[23] Tetsushi Nishida and Kokichi Sugihara, Precision necessary for $d$-dimensional Sphere Voronoi Diagrams, Proceedings of the Fifth International Symposium on Voronoi Diagrams in Science and Engineering (ISVD 2008), 2008, pp. 157-167.

[24] Tetsushi Nishida, Yoshiaki Tanaka, and Kokichi Sugihara, Evaluation of the Precision for Exact Computation of a Circle Voronoi Diagram, Tech. Report UW-CS-TR-1481, Department of Mathematical Informatics, Graduate School of Information Science and Technology, The University of Tokyo, Bunkyo-Ku, Tokyo 113-8656, Japan, October 2007.

[25] Atsuyuki Okabe, Barry Boots, and Kokichi Sugihara, Spatial tessellations: Concepts and applications of voronoi diagrams, John Wiley and Sons, 1992.

[26] Atsuyuki Okabe, Barry Boots, and Kōkichi Sugihara, Spatial tessellations: concepts and applications of Voronoï diagrams, John Wiley \& Sons Ltd., Chichester, 1992, With a foreword by D. G. Kendall. MR 94a:52033

[27] Joonghyun Ryu, Donguk Kim, Youngsong Cho, Rhohun Park, and Deok soo Kim, D-s, computation of molecular surface using euclidean voronoi diagram, Computer-Aided Design and Applications, Vol.2, Nos. 1-4, 2005.

[28] Deok soo Kim, Donguk Kim, Youngsong Cho, and Kokichi Sugihara, Quasi-triangulation and interworld data structure in three dimensions, Computer-aided Design 38 (2006), 808-819.

[29] G. F. Voronoï, Nouvelles applications des paramètres continus à la théorie des formes quadratiques. premier mémoire. sur quelques propriétés des formes quadratiques positives parfaites., Journal für die reine und angewandte Mathematik 133 (1907), 97-178.

[30] _ Nouvelles applications des paramètres continus à la théorie des formes quadratiques. deuxième mémoire. recherches sur les paralléloèdres primitifs. première partie. partition uniforme de l'espace analytique à $n$ dimensions à l'aide des translations d'un même polyèdre convexe., Journal für die reine und angewandte Mathematik 134 (1908), 198-287.

[31] Nouvelles applications des paramètres continus à la théorie des formes quadratiques. deuxième mémoire. recherches sur les paralléloèdres primitifs. seconde partie. domaines de formes quadratiques correspondant aux différents types de paralléloèdres primitifs., Journal für die reine und angewandte Mathematik 136 (1910), 67-181.

[32] Hans-Martin Will, Fast and efficient computation of additively weighted voronoi cells for applications in molecular biology, Proceedings of the 6th Scandinavian Workshop on Algorithm Theory (London, UK), SWAT '98, Springer-Verlag, 1998, pp. 310-321.

[33] Wen-Tsun Wu and Xiao-Shan Gao, Automated reasoning and equation solving with the characteristic set method, J. Comput. Sci. \& Technol. 21 (2006), no. 5, 756-764. 